\title{
Response familiarization and subsequent paired-associate learning for mixed and unmixed lists of differential meaningfulness
}

\author{
D. GENE DAVENPORT, DONALD H. KAUSLER, JOHN H. MUELLER, JR. AND ALGIMANTAS A. NORVILAS \\ SAINT LOUIS UNIVERSITY
}

Two experiments, one with lists containing items of mixed meaningfulness and the other with lists of unmixed meaningfulness, related response familiarization, via free recall learning, to subsequent paired-associate learning. Both relevant and irrelevant familiarization were superior to no familiarization, but the advantage of relevant over irrelevant familiarization was limited to responses of low meaningfulness only and to the unmixed condition only.

Relevant response familiarization, relative to irrelevant and no familiarization, is expected to facilitate subsequent paired-associate (PA) learning through the savings engendered in the $\mathrm{PA}$ response learning stage (Underwood \& Schulz, 1960). Similarly, irrelevant response familiarization should promote more proficient PA learning than no prior familiarization through the transfer of nonspecific factors. Nevertheless, as emphasized in the recent review by Goss \& Nodine (1965)。 experiments on response familiarization have ylelded inconsistent and conflicting results in terms of these expected facilitative effects. The need for further empirical evidence is clearly indicated.

The present study consisted of two experiments in which familiarization was accomplished by the free recall learning of items of differential meaningfulness (M). A mixed list containing items of low (L), medium (Md), and high $(\mathrm{H}) \mathrm{M}$ was employed in Experiment 1. The advantage of relevant over irrelevant familiarization was expected to be most pronounced for the L M items and least pronounced for the H M items. However, PA learning for the relevant and irrelevant groups was not found to differ, although both groups were clearly absent. Viewing the results of Experiment 1 as being potentially confounded by the use of a mixed list, the relevant and irrelevant conditions were repeated in Experiment II with unmixed lists of homogeneous L M and homogeneous $\mathrm{H}$ M items.

Method

The $45 \mathrm{Ss}$ in Experiment I were assigned alternately to three groups ( $N, R$, and $I ; N=15)$. All three groups practiced on the same $P A$ list to a criterion of one perfect trial. The PA list was composed of nine single digit-nonsense syllable pairs, with three of the response items being $\mathrm{L} M(0-20 \%$ Archer (1960) values, $3 \mathrm{Md} \mathrm{M}$ $(40-60 \%)$, and $3 \mathrm{H} \mathrm{M}(80-100 \%))$. Group $N$ received no prior familiarization training; Group $\mathrm{R}$ received prior training on relevant response items (i.e., the specific response items of the $\mathrm{PA}$ list), and Group I received familiarization training on irrelevant items (i.e., items comparable in $M$ values to those of the PA list but unrelated in content). Familiarization training was by free recall learning to a criterion of three successful perfect trials. Intralist and interlist (for Group I) similarity of response items was minimized as much as possible. For free recall the items were presented at a 2-sec. rate; a 45-sec. recall period followed each study trial. PA practice was by the aniticipation method at a 2-2 sec. rate. Three different serial orders were employed across trials for both free recall and PA practice as a control for serial learning effects. All learning materials were presented by means of a Carousel projector.

The 60 Ss of Experiment II were assigned alternately to the four conditions of a 2 by 2 factorial representing two levels of $M(L$ and $H$ ) and either relevant or irrelevant familiarization (Groups $R L, R H, I L$, and $I H ; N=15$ ). Except for the use of unmixed lists and the exclusion of a no familiarization condition, the procedure of this experiment closely replicated that of Experiment I. Results and Discussion

Experiment I. Groups R and I were comparable in their free recall performance (mean number of trials to criterion $=13.8$ and 12.4 , respectively; $t<1, d f=28$ ). For trials to criterion on the PA list the mean and standard deviation were 15.27 and $5.48,8.80$ and 3.12 , and 9.27 and 3.84 for Groups $\mathrm{N}, \mathrm{R}$, and I, respectively. Although an analysis of variance revealed an overall significant between-group effect for PA trials $(F=10.72, \mathrm{df}=2 / 42$, $p<.001$ ), Duncan's multiple range test indicated that the effect was attributable only to the inferior performance of Group $N$ relative to both Group R and Group I (both ps $<.005)$. The Group R vs. Group I comparison did not approach significance. In addition, total errors to criterion were determined separately for each level of $\mathrm{M}$; as well as for each familiarization condition, and were analyzed by a 2 by 2 mixed analysis of variance. The between-group familiarization effect was highly significant $(F=18.42, \mathrm{df}=2 / 42, \mathrm{p}<.001)$, with the effect again being attributable solely to the inferior performance of Group $\mathrm{N}$ relative to Groups $\mathrm{R}$ and I. The withingroup $M$ effect was also highly significant $(F=8.31$, $\mathrm{df}=2 / 84, \mathrm{p}<.001$ ), with the mean number of errors being $18.05,17.67$, and 12.87 for $L, M d$, and $H M$ pairings, respectively. However, the familiarization by $M$ interaction was clearly not significant ( $F<1$, df $=4 / 84$ ).

The above results suggest that irrelevant response familiarization is as effective as relevant familiarization in promoting subsequent $\mathrm{PA}$ acquisition and that 
the effect is relatively independent of the M level of the response items. The positive effect of irrelevant familiarization most likely represents pronounced nonspecific transfer. Relevant familiarization should contribute a pronounced specific transfer in addition to the nonspecific transfer, presumably equal to that of irrelevant familiarization. The failure to find an advantage of relevant over irrelevant familiarization implies the possibility of an additional negative transfer component present in the relevant condition only that reduces the overall resultant positive transfer. This negative component may be the consequence of the use of a mixed M list. Schulz \& Thysell (1965) recently reported that familiarization with a mixed M list increases the number of intralist associations given to the items as stimuli, with the effect being most pronounced for $\mathrm{L} M$ stimuli. Conceivably such intralist associations interfere with PA associative learning in the relevant condition.

Experiment II. The mean number of trials to the free recall criterion were $18.66,12.86,15.86$, and 15.26 for Groups $\mathrm{RL}, \mathrm{RH}, \mathrm{IL}$, and IH, respectively. The analysis of variance indicated that only the effect of $M$ approached significance $(F=2.84, \mathrm{df}=1 / 56, \mathrm{p}<.10)$. For $\mathrm{PA}$ trials to criterion the means and standard deviations were 8.26 and 2.96, 7.46 and 2.10, 11.40 and 5.84, and 8.46 and 4.81 for the same ordering of groups. The $M$ and familiarization effects both approached significance ( $F s=2.97$ and $3.64, \mathrm{df}=1 / 56, \mathrm{p}<.10$ ), but the interaction effect was negligible $(F<1)$. For total errors the means and standard deviations were 22.76 and 12.70 , 25.20 and $10.60,37.40$ and 33.02 , and 28.53 and 15.88, for RL, RH, IL, and IH conditions. The effects of both $M$ and familiarization were significant ( $F s=6.04$ and 4.62, df $=1 / 56, \mathrm{ps}<.05$; square root transformation with heterogeneity of variance). Although the interaction effect was again nonsignificant $(p>.10)$, a Duncan's test revealed that the effects of $\mathrm{M}$ and familiarization were attributable only to the inferior performance of Group IL contrasted with the other three groups (all ps $<.05$ ). None of the other comparisons approached significance. Duncan's test revealed a similar pattern of contrasts for trials to criterion. These comparisons imply that the advantage of relevant response familiarization over irrelevant is limited to response items of low $\mathrm{M}$-an implication that is consistent with the tenets of stage analysis.

\section{References}

Archer, E. J. A re-evaluation of the meaningfulness of all possible CVC trigrams. Psychol. Monogr., 1960, 74, No. 10 (Whole No. 497).

Goss, A. E., \& Nodine, C. F. Paired-associates learning. New York: Academic Press, 1965.

Schulz, R. W., \& Thysell, R. The effect of familiarization on meanfulness. J. verbal Learn. verbal Behav., 1965, 4, 409-413.

Underwood, B. J., \& Schulz, R. W. Meaningfulness and verbal learning. Chicago: Lippincott, 1960. 